\title{
A 3-state defect model for light-induced degradation in boron-doped float-zone silicon
}

\author{
David Sperber ${ }^{*}$, Axel Herguth, and Giso Hahn \\ Department of Physics, University of Konstanz, Universitätsstr. 10, 78464 Konstanz, Germany
}

Keywords float-zone silicon, light-induced degradation, charge carrier lifetime, defects, boron, doping

* Corresponding author: e-mail david.sperber@uni-konstanz.de, Phone: +49 7531885242

We report on a light-induced bulk defect activation and subsequent deactivation in boron doped float-zone silicon that can be described by a 3-state model. During treatment at elevated temperature and illumination, a sample first converts from an initial high lifetime state into a degraded low lifetime state and then shows a recovery reaction leading to a third high lifetime state that is then stable under degradation conditions. Furthermore, it is shown that reverse reactions into the initial state appear to be possible both from the degraded as well as the regenerated state. An injection dependent analysis of lifetime data yields a defect capture cross section ratio of $\sim 20$ suggesting a positively charged defect.
1 Introduction Light-induced degradation (LID) may severely limit the bulk charge carrier lifetime $\tau_{b}$ in crystalline silicon [1] which poses a problem to devices relying on stable high $\tau_{b}$ such as sensors or solar cells. Examples are LID in multicrystalline (mc) silicon [2-4] and boronoxygen (BO) related degradation in Czochralski-grown (Cz) monocrystalline silicon [5]. Float-zone (FZ) silicon, on the other hand, is usually supposed to be characterized by a stable and high $\tau_{b}$. However, reports about instabilities of $\tau_{b}$ in FZ silicon are becoming more frequent [6-8].

In this study, a degradation and recovery of $\tau_{b}$ is reported in boron-doped FZ samples treated at elevated temperature and illumination. It will be shown that a 3-state defect model, as used e.g. for BO degradation, appears to be a promising approach for the description. In addition, evidence for different reaction pathways between the three states, particularly reverse reaction paths, is provided.

2 Experimental All samples shown here were made out of a single boron doped FZ wafer with specific resistivity $2 \Omega \mathrm{cm}$, diameter $150 \mathrm{~mm}$, and thickness $250 \mu \mathrm{m}$. The wafer first received a cleaning in a solution of $\mathrm{H}_{2} \mathrm{O}_{2}$ and $\mathrm{H}_{2} \mathrm{SO}_{4}(1: 4)$ at $80{ }^{\circ} \mathrm{C}$ followed by a dip in HF. Afterwards, the wafer was split in halves which were coated with hydrogenated silicon nitride $\left(\operatorname{SiN}_{x}: \mathrm{H}\right)$ on both sides. Finally, both halves received a short high temperature step in a fast-firing belt furnace at a measured peak sample temperature of $\sim 835^{\circ} \mathrm{C}$ to activate the passivation. Samples of edge length $5 \mathrm{~cm}$ were cut out using a laser and stored in darkness until sample treatment began.

The samples were treated on hotplates either in darkness or at an illumination intensity of 1 sun equivalent. For the repeated measurement of effective lifetime $\tau_{\text {eff }}$ in dependence of injection level $\Delta n$, the sample treatment was shortly paused and a measurement of the photoconductance decay (PCD) was carried out using a Sinton Instruments lifetime tester (WCT-120) at $30^{\circ} \mathrm{C}$. All lifetime data were corrected for Auger and radiative recombination according to the parameterization of Richter et al. [9].

\section{Results}

3.1 Evolution of effective lifetime As can be seen in Fig. 1(a), a sample treated at $80^{\circ} \mathrm{C}$ and $\sim 1$ sun equivalent illumination intensity shows a strong degradation leading to a minimum of $\tau_{\text {eff }}$ after $\sim 1 \mathrm{~h}$. For longer treatment times, a recovery of $\tau_{\text {eff }}$ can be observed. A combination of corona charging series, capacitance voltage measurements and repassivation of the sample surface in similarly processed samples leads to the conclusion that the degradation is caused in the bulk of a sample. These measurements are 


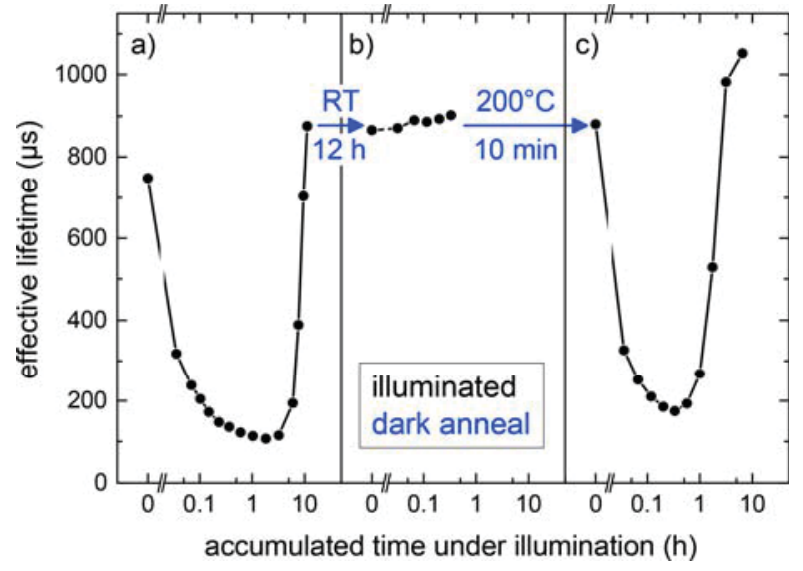

Figure 1 Measurement of $\tau_{\text {eff }}$ during treatment at $80^{\circ} \mathrm{C}$ and $\sim 1$ sun equivalent illumination intensity (a) starting with a fresh sample, (b) after storage in darkness at room temperature (RT) over night, and (c) after $10 \mathrm{~min}$ at $200{ }^{\circ} \mathrm{C}$ in darkness. All measurements were evaluated at an injection level of $\Delta n=7 \times$ $10^{14} \mathrm{~cm}^{-3}$. Lines only serve as guide to the eye.

thoroughly discussed in a separate publication [10] whereas the scope of the study at hand is to further analyze the bulk degradation.

According to Fig. 1(b), after storage in darkness over night at room temperature, no degradation is observed during an identical illuminated treatment at $80^{\circ} \mathrm{C}$ but the sample continues its recovery. However, annealing in darkness at $200{ }^{\circ} \mathrm{C}$ for $10 \mathrm{~min}$ and subsequent treatment at $80^{\circ} \mathrm{C}$ and illumination (as in the previous steps) leads again to a degradation within minutes (c). This degradation is similar to (a) but slightly weaker and the recovery sets in earlier.

The treatment of another sample was interrupted before reaching the minimum (and subsequent recovery) in $\tau_{\text {eff }}$ as shown in Fig. 2(a). The sample was then annealed in darkness at $200{ }^{\circ} \mathrm{C}$ for $5 \mathrm{~min}$. As can be seen in Fig. 2(b), $\tau_{\text {eff }}$ is close to the initial value after the annealing step and the sample degrades again when subjected to $80^{\circ} \mathrm{C}$ and illumination. According to Fig. 2(c), this procedure can be repeated. The values of $\tau_{\text {eff }}$ show, however, a small decrease after each additional cycle that could not be reversed by a doubled annealing time of $10 \mathrm{~min}$ after (c). Comparing surface saturation current density $\left(J_{0 \mathrm{~s}}\right)$ values at $t=0 \mathrm{~h}$ shows that the small decreases are probably caused by a degradation of surface passivation quality [11].

3.2 Injection dependent analysis One possible cause of light-induced degradation is a contamination with iron resulting in $\mathrm{FeB}$ pairing in darkness and $\mathrm{FeB}$ dissociation under illumination [12]. However, FeB degradation does not seem to be an issue in our samples because illumination after some days of storage in darkness did not cause significant changes (especially no crossover in low injection) neither in an untreated nor in a partially degraded sample.

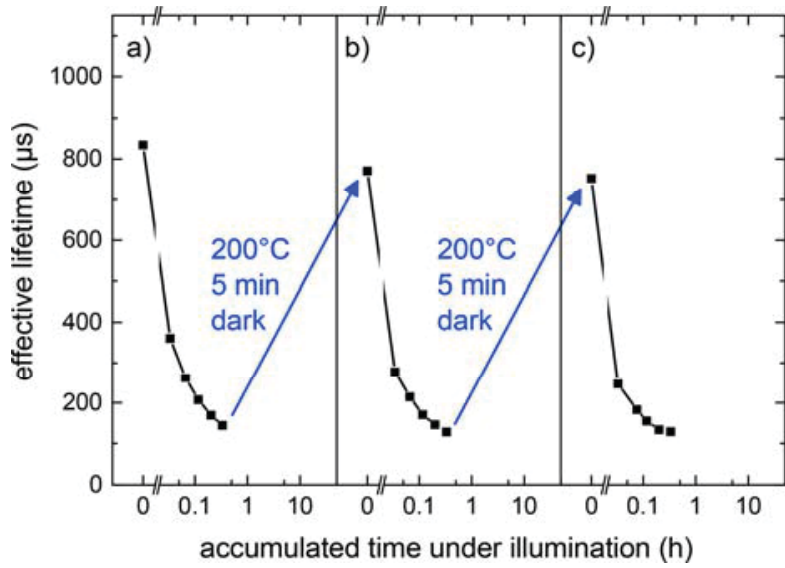

Figure 2 Measurement of $\tau_{\text {eff }}$ during treatment at $80{ }^{\circ} \mathrm{C}$ and $\sim 1$ sun equivalent illumination intensity (a) starting with a fresh sample, (b) after $5 \mathrm{~min}$ at $200{ }^{\circ} \mathrm{C}$ in darkness, and (c) after another $5 \mathrm{~min}$ at $200{ }^{\circ} \mathrm{C}$ in darkness. All measurements were evaluated at an injection level of $\Delta n=7 \times 10^{14} \mathrm{~cm}^{-3}$. Lines only serve as guide to the eye.

Figure 3 shows the changing injection dependency of $\tau_{\text {eff }}$ during the treatment shown in Fig. 1(a). Both before treatment and after long treatment times, the injection dependency is rather flat. After $1 \mathrm{~h}$ of treatment, however, $\tau_{\mathrm{eff}}$ is strongly decreased, especially in low injection.

$\tau_{\text {eff }}$ can be described by the sum of a time dependent defect concentration $N_{\text {def }}(t)$ and a constant defect background lifetime $\tau_{\mathrm{bg}}$ :

$$
\frac{1}{\tau_{\mathrm{eff}}(t)}=c \cdot N_{\mathrm{def}}(t)+\frac{1}{\tau_{\mathrm{bg}}} .
$$

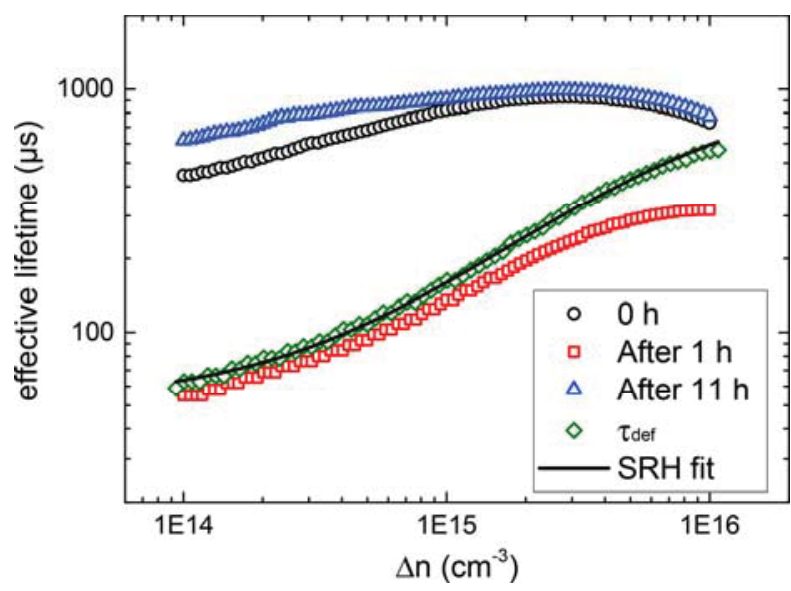

Figure 3 Changes in injection dependence of $\tau_{\text {eff }}$ during treatment at $80^{\circ} \mathrm{C}$ and $\sim 1$ sun equivalent illumination intensity. Measurement data are taken from the treatment shown in Fig. 1(a). They are already corrected by Auger and radiative recombination according to [9] and therefore only reflect recombination via defect levels in the bulk or at the surface. The values of $\tau_{\text {def }}$ (green) were calculated by comparing data from the untreated (black) and degraded (red) state. Subsequently, a SRH fit of $\tau_{\text {def }}$ was conducted and is shown as a black line. 
A comparison of $\tau_{\text {eff }}$ at two different times of treatment yields the corresponding change in defect concentration $\Delta N_{\text {def }}$ and can be used to define a defect related lifetime $\tau_{\text {def }}$ as follows:

$$
\frac{1}{\tau_{\mathrm{def}}(t)}:=c \cdot \Delta N_{\mathrm{def}}(t)=\left(\frac{1}{\tau_{\mathrm{eff}}(t)}-\frac{1}{\tau_{\mathrm{eff}}\left(t_{0}\right)}\right) .
$$

We use the theory of Shockley, Read and Hall (SRH) $[13,14]$ under the assumption of a single deep defect level to determine the characteristic capture cross section ratio $\sigma_{n} / \sigma_{p}$ of electrons and holes. A fit of $\tau_{\text {def }}$ resulting from the data before and after $1 \mathrm{~h}$ of treatment as shown in Fig. 3 yields a $\sigma_{n} / \sigma_{p}$ ratio of $\sim 20$, suggesting a positively charged defect in our FZ samples. This value was calculated assuming stable surface passivation and stable defect background. This seems to be justified because the comparison of data at different treatment times (up to $3 \mathrm{~h}$ ) either with data before treatment (black) or after recovery (blue) leads to a rather constant $\sigma_{n} / \sigma_{p}$ ratio of $20 \pm 2$.

4 Discussion For defects that express both degradation and recovery at the same treatment conditions, a 3 -state defect model as proposed in Fig. 4 is a promising approach to explain the observed behaviour.

A prominent example of a 3-state model is the one used in the description of $\mathrm{BO}$ related degradation in $\mathrm{Cz}$ silicon [15]. In this model, a defect evolves from an annealed state A into a degraded state B and subsequently recovers into a supposedly stable regenerated state $\mathrm{C}$ when subjected to appropriate temperature and illumination conditions. The charge carrier lifetime of the macroscopic sample is then defined by a defect pool in which different defect states are occupied to a certain degree. For example, a partially degraded sample refers to a mixture of some defects being in state A and some in state B. The continuous change in lifetime corresponds to a redistribution within the defect pool.

Additionally, it is known that reverse reactions from $\mathrm{B}$ to $\mathrm{A}$ and from $\mathrm{C}$ to $\mathrm{A}$ (possibly via $\mathrm{B}$ ) occur during elevated temperature treatment and become observable espe-

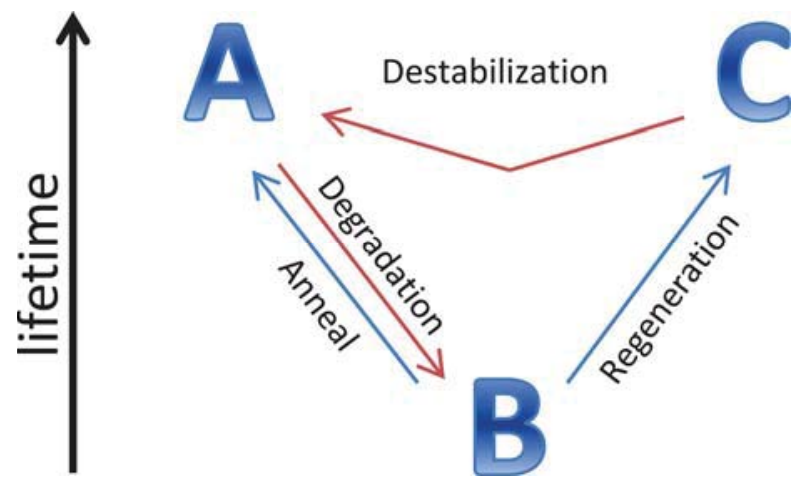

Figure 4 Illustration of the proposed 3-state defect model and associated reaction pathways for charge carrier lifetime degradation and regeneration in boron-doped FZ silicon. cially in darkness when other reaction pathways are inactive. The FZ samples in the present study have been subjected to similar temperature and illumination treatments and possible reaction pathways can now be identified.

From Fig. 2(a) it can be concluded that non-degraded samples (state A, high lifetime) evolve towards a degraded state B (low lifetime) when subjected to an elevated temperature and illumination treatment. A temperature step at $200{ }^{\circ} \mathrm{C}$ in darkness seems to lead back to the non-degraded state A, because afterwards a sample degrades very similarly compared to an untreated sample. If treated at elevated temperature and illumination for a longer time, however, a regeneration reaction occurs as seen in Fig. 1(a), leading towards a regenerated state $\mathrm{C}$ (high lifetime).

According to Fig. 1(b), state $\mathrm{C}$ appears to be rather stable during a continued treatment. Subjecting a sample in state $\mathrm{C}$ to a treatment at $200{ }^{\circ} \mathrm{C}$ in darkness, however, partially destabilizes a regenerated sample, bringing it back towards state A: it degrades again starting from a high lifetime level comparable to the untreated sample when subjected to an elevated temperature and illumination treatment as shown in Fig. 1(c). The slightly weaker degradation amplitude and faster recovery in Fig. 1(c) could be related to an incomplete destabilization due to a rather short annealing time at $200{ }^{\circ} \mathrm{C}$. It cannot be ruled out that the transition from state $\mathrm{C}$ towards state $\mathrm{A}$ corresponds to a two-step reaction via state $\mathrm{B}$.

It is interesting to note that the defect under investigation in FZ silicon reacts to very similar treatment conditions compared to $\mathrm{BO}$ defects in $\mathrm{Cz}$ silicon. It is nevertheless unlikely that the cause of degradation is the same. On the one hand, $\sigma_{n} / \sigma_{p}$ is known to have a value of $\sim 10$ for the slowly forming BO defect and a value of $\sim 100$ for the fast forming defect center [16] which do both differ significantly from the value of $\sim 20$ determined in our FZ samples.

Additionally, it has been shown in great detail that the strength of BO degradation depends strongly on the oxygen content of a sample. The upper limit of oxygen concentration in the FZ material used here is given by the supplier as $10^{16} \mathrm{~cm}^{-3}$. This would lead to a BO degraded lifetime of around $70 \mathrm{~ms}$ for $2 \Omega \mathrm{cm}$ material according to the parameterization of Bothe et al. [17] that was determined using $\mathrm{Cz}$ silicon, far too high to explain the observed lifetime changes in FZ silicon. It appears, however, possible that regeneration of our FZ samples and regeneration of BO degraded samples share a common mechanism. This, e.g., could be a hydrogen passivation of the defect which is often suspected in BO regeneration [18, 19].

The defect discussed here could be related to a defect described by Grant et al. [6] that occurs in as-grown p-type but not in n-type FZ silicon. Their value of $\sigma_{n} / \sigma_{p}$ of $\sim 26$ is rather close to our value of $\sim 20$ and the observations in [6] agree well with transitions between states A and B. However, a state $\mathrm{C}$ has not been described in the cited study. This could be related to different passivation methods used which might change sample properties. E.g., our samples are expected to feature a rather high hydrogen content due 
to the $\operatorname{SiN}_{x}: \mathrm{H}$ layer and the fast firing step whereas the samples in [6] should rather have a low hydrogen content. Consequently, a hydrogen passivation of the defect appears to be possible in our samples whereas it might not be possible in the samples described in [6].

5 Conclusion In this study, a degradation and subsequent recovery of bulk lifetime $\tau_{b}$ was observed in borondoped float-zone silicon during treatment at elevated temperature and illumination. This was attributed to a defect switching from an initial state A into a degraded state B and subsequently into a regenerated state C. Additionally, it has been shown that reverse reactions seem to occur during a temperature treatment in darkness. These reverse reactions to the initial state $\mathrm{A}$ appear to be possible both from the degraded state B as well as from the regenerated state C. An injection dependent analysis was used to differentiate the defect from $\mathrm{FeB}$ and $\mathrm{BO}$ related degradation and the capture cross section ratio $\sigma_{n} / \sigma_{p}$ was determined to be $\sim 20$, suggesting a positively charged defect.

Acknowledgements The authors would like to thank A. Graf, A. Heilemann, L. Mahlstaedt, B. Rettenmaier, S. Joos, and J. Engelhardt for technical support. Part of this work was supported by the German Federal Ministry for Economic Affairs and Energy under contract numbers 0325763B, 0325581 and 0324001 . The content is the responsibility of the authors.

\section{References}

[1] J. Lindroos and H. Savin, Sol. Energy Mater. Sol. Cells 147, 115 (2016)

[2] K. Ramspeck, S. Zimmermann, H. Nagel, A. Metz, Y. Gassenbauer, B. Birkmann, and A. Seidl, in: Proc. 27th Eur.
Photovoltaic Sol. Energy Conf. Exhib., Frankfurt/Main, Germany, 2012, pp. 861-865.

[3] F. Fertig, K. Krauß, and S. Rein, Phys. Status Solidi RRL 9, 41 (2015).

[4] F. Kersten, P. Engelhart, H. C. Ploigt, A. Stekolnikov, T. Lindner, F. Stenzel, and J. W. Müller, Sol. Energy Mater. Sol. Cells 142, 83 (2015).

[5] K. Bothe and J. Schmidt, J. Appl. Phys. 99, 13701 (2006).

[6] N. E. Grant, F. E. Rougieux, D. Macdonald, J. Bullock, and Y. Wan, J. Appl. Phys. 117, 55711 (2015).

[7] N. E. Grant, V. P. Markevich, J. Mullins, A. R. Peaker, F. Rougieux, and D. Macdonald, Phys. Status Solidi RRL 10, 443 (2016)

[8] F. E. Rougieux, N. E. Grant, C. Barugkin, D. Macdonald, and J. D. Murphy, IEEE J. Photovolt. 5, 495 (2015).

[9] A. Richter, S. W. Glunz, F. Werner, J. Schmidt, and A. Cuevas, Phys. Rev. B 86, 165202 (2012).

[10] D. Sperber, A. Heilemann, A. Herguth, and G. Hahn, IEEE J. Photovolt., accepted, DOI 10.1109/JPHOTOV.2017.2649601.

[11] K. R. McIntosh and L. E. Black, J. Appl. Phys. 116, 014503 (2014).

[12] D. Macdonald, J. Tan, and T. Trupke, J. Appl. Phys. 103, 073710 (2008)

[13] W. Shockley and W. T. Read Jr., Phys. Rev. 87, 835 (1952).

[14] R. N. Hall, Phys. Rev. 87, 387 (1952).

[15] A. Herguth and G. Hahn, J. Appl. Phys. 108, 114509 (2010).

[16] K. Bothe and J. Schmidt, Appl. Phys. Lett. 87, 262108 (2005).

[17] K. Bothe, R. Sinton, and J. Schmidt, Prog. Photovolt. 13, 287 (2005).

[18] S. Wilking, A. Herguth, and G. Hahn, J. Appl. Phys. 113, 194503 (2013).

[19] N. Nampalli, B. Hallam, C. Chan, M. Abbott, and S. Wenham, Appl. Phys. Lett. 106, 173501 (2015). 